\title{
Non-adherence to topical treatments for actinic keratosis
}

\author{
Bav Shergill' \\ Simon Zokaie ${ }^{2}$ \\ Alison J Carr ${ }^{3}$ \\ 'Department of Dermatology, \\ Brighton and Sussex University \\ Hospitals, Elm Grove, Brighton, UK; \\ ${ }^{2}$ Leo Pharma, Princes Risborough, \\ ${ }^{3}$ Hamell, London, UK
}

This article was published in the following Dove Press journal:

Patient Preference and Adherence

16 December 2013

Number of times this article has been viewed

Background: There is limited information on the patterns of use, adherence rates, and factors that impact adherence with topical treatments for actinic keratosis (AK).

Objectives: To establish patterns of use and adherence with topical treatments for AK and to identify treatment-related factors that impact on adherence.

Methods: A community-based, cross-sectional study was performed using a standardized questionnaire completed online or via telephone interview. Patients were stratified according to the presence of AK lesions on the scalp and/or other extremities; and presence of scarring resulting from treatment.

Results: This study included 305 patients with AK who were currently using a patient-applied topical therapy for AK or had used one within the previous 12 months. In total, 88\% ( $n=268 / 305)$ of patients were either non-adherent, non-persistent or both non-adherent and non-persistent to topical therapy. Duration of treatment was associated with increasing rates of non-adherence (adjusted odds ratio [OR]; for treatment durations greater than 4 weeks, 2.2, $P<0.01$ ): 52\% of patients were non-adherent with 3-4 week treatment duration; $69 \%$ of patients with 4-8 week treatment duration; and $71 \%$ of patients with 6-12 week treatment duration. There were similar increases in non-persistence with increasing treatment duration (adjusted OR; for treatment durations greater than 4 weeks, $2.1, P<0.05$ ).

Conclusion: This study found high rates of non-adherence and non-persistence in patients with AK. Duration of treatment was a significant factor contributing to non-adherence and non-persistence to topical treatments. Patient-applied topical therapies that require less frequent application and have shorter treatment duration may be associated with improved adherence rates.

Keywords: patient compliance, chronic skin disease, treatment duration, patient behavior

\section{Introduction}

Actinic, or solar, keratoses (AKs) are common skin lesions that affect approximately $20 \%$ of people over 60 years of age in the UK. ${ }^{1}$ AKs are generally the result of cumulative ultraviolet skin exposure and are precursors for the majority of cutaneous squamous cell carcinoma (SCC) cases. With an aging population, increase in outdoor activities, and changes in sunbathing practices, the incidence of $\mathrm{AKs}$ is increasing in older persons as well as those aged younger than 40 years. ${ }^{2}$ In general, AKs occur predominantly on sun exposed areas: the backs of the hands, the face, scalp, and forehead. They are often asymptomatic but may occasionally be sore or itch. Patients often have multiple lesions within a field of damage. ${ }^{3}$ Over time, AK lesions may remain unchanged, proliferate, or regress. Approximately $25 \%$ of lesions recede without treatment. ${ }^{1,2}$ Although the
Correspondence: Alison J Carr Hamell, The Loft, Ia Salcott Road, London SWII 6DQ, UK

Tel + 4402079785206

Email alison@hamell.co.uk 
rate of progression of AKs to SCC is relatively low (around $8 \%$ on average $\mathrm{e}^{4}$ ), the main reason that treatment is initiated is to prevent progression into SCC. ${ }^{1}$

The two main approaches to treating AK lesions are patient-applied topical therapies which are applied to single lesions, multiple lesions, or an entire area or field, ${ }^{2}$ and surgical or ablative methods (eg, cryotherapy) targeted to one lesion. Although new topical agents are in the early stages of being introduced, the mainstays of topical treatment are currently: diclofenac sodium 3\% gel, 5-fluorouracil 5\% cream, imiquimod $5 \%$ cream, and fluorouracil $5 \mathrm{mg} / \mathrm{g}$ and salicylic acid $100 \mathrm{mg} / \mathrm{g}$ solution. ${ }^{1}$ These topical agents vary in terms of their efficacy, cost, cosmetic acceptability, side effects, and frequency and duration of treatment. All agents must be applied for at least several weeks and typically cause some erythema, burning, and ulceration at the application site. The British Association of Dermatologists Guidelines for the Management of Actinic Keratoses (2007) ${ }^{1}$ state that data comparing individual treatments are not sufficient to justify making a single recommendation. They advise that treatment decisions should be individualized and based on clinical presentation, product characteristics, and patient preference.

For self-administered treatments, adherence is another factor to be considered when selecting a suitable treatment for patients with AKs. Adherence to patient-applied topical therapy regimens has been found to be low for a range of skin conditions, especially chronic skin disorders such as psoriasis which require long-term treatment. ${ }^{5,6}$ Approximately $40 \%$ of patients with psoriasis report not using treatments as directed. ${ }^{7}$ One study in a small number of patients with AKs reported higher levels of adherence to a once-a-day topical therapy (around 80\% averaged over the 4-week treatment period), although rates of adherence declined over the treatment period. ${ }^{8}$ Generalizability of the results of this study are limited by the very small sample size ( 20 patients), and the exclusion of lesions on parts of the body other than the face and scalp.

Treatment non-adherence has been associated with poor response to treatment and worse disease outcomes. ${ }^{5,6}$ Non-persistence (not completing the course of treatment) can be viewed as another type of non-adherence. It is possible for patients to be non-adherent (not use the correct dose at the correct times) but still complete the course of treatment, and therefore be persistent. Conversely, patients can use the correct dose at the correct times (and therefore be adherent) but stop the course of treatment prematurely and therefore be non-persistent. Non-adherence can be unintentional (forgetting doses) or based on logical, reasoned decisions about how and when to use medication (intentional non-adherence). Most interventions have traditionally been aimed at reducing unintentional non-adherence using treatment reminders and prompts. However, in general, forgetting doses probably accounts for only around $40 \%$ of non-adherence. As highlighted in previous research, nonadherence to patient-applied topical treatments is influenced by sociodemographic, disease, and treatment-related factors. Some of these factors include younger age, ${ }^{5,9}$ forgetfulness, ${ }^{10}$ patient satisfaction, ${ }^{9,11}$ perceptions of treatment efficacy, ${ }^{11}$ lack of knowledge, ${ }^{9}$ having to pay for prescriptions, ${ }^{10}$ the cosmetic characteristics of treatment, ${ }^{5}$ the complexity of the treatment regimen, ${ }^{11}$ frequency of use, ${ }^{12}$ and treatment duration. ${ }^{12}$ Some factors contributing to non-adherence including inconvenience, ${ }^{11}$ time constraints, ${ }^{5,12}$ and fear of, ${ }^{11}$ or occurrence of, side effects ${ }^{9}$ are common to many skin disorders.

This study aimed to explore how patients with AK use their topical treatments (patterns of use). The objective was to determine the rates of adherence and persistence with different patient-applied topical therapies for AK in a large sample of patients with lesions on all parts of the body; and the extent to which adherence and persistence are influenced by treatment characteristics including the complexity and duration of treatment regimens.

\section{Materials and methods}

This was a community-based cross-sectional study in patients with AK from across the UK. The study was conducted in May 2012. Patients were recruited online and via adverts in local and national newspapers.

Patients completed a questionnaire online or via telephone interview. This questionnaire was based on standardized questionnaires used in previous studies to capture patterns of medication use and identify factors associated with poor adherence and non-persistence. ${ }^{13,14}$ The protocol, questionnaire, and other study materials were reviewed and approved by an independent research ethics committee. All subjects provided informed consent.

Eligible patients were adults ( $>18$ years old) with a clinical diagnosis of AK made by their GP or specialist, affecting the head and/or trunk and extremities, who were currently or had previously (within the last 12 months) used one of the following patient-applied topical treatments for AK: diclofenac sodium 3\% gel, 5-fluorouracil 5\% cream, imiquimod $5 \%$ cream, or fluorouracil $5 \mathrm{mg} / \mathrm{g}$ and salicylic acid $100 \mathrm{mg} / \mathrm{g}$ solution. Patients who had previously 
received cryotherapy or excision surgery for AK lesions were excluded from the study. The sample was stratified according to whether subjects were currently using treatment or had ceased treatment within the previous 12 months. The sample was also stratified to ensure the study included comparable numbers of subjects with AK affecting the head and other body sites (trunk and extremities), and comparable proportions of subjects whose treatment had and had not resulted in scarring.

Data collected included: sociodemographic characteristics (sex, geographic region, ethnicity, marital status, total annual household income, highest level of education, and employment status) and disease characteristics (time since initial diagnosis of AK; current or most recent topical AK treatment used; time since last topical treatment for AK was used; presence of scarring; and current or previous localized skin reaction [LSR]).

Patient-reported measures of adherence consistently overestimate adherence compared with more objective measures such as pill counts, tube weights, and electronic pill caps. ${ }^{15-22}$ To reduce this bias, patients were asked to give detailed information about how they used their cream/ ointment (frequency of application, times when application was missed or forgotten, length of time [days/weeks] treatment was used) without reference to how frequently they should have been/had been advised to use it. These questions allowed patients to give information about medication use in a more factual, less biased way than if they had been asked whether they had followed their doctor's recommendation. Estimates of adherence and persistence were calculated from these data. Once they had given this information patients were asked how often they forgot to apply the cream/ointment to gain an estimate of unintentional non-adherence. Quality of life was measured using a validated dermatology-specific questionnaire, Skindex $16 .^{23}$ Hypothetical treatment scenarios incorporating variations in efficacy and treatment duration were used to elicit subjects' treatment preferences.

A sample of 300 patients with AK was required for the logistic regression where non-adherence was categorized as a binary variable, to detect an odds ratio of two in the explanatory variable, with $80 \%$ power, and $5 \%$ significance.

Recruitment methods used in this study helped to minimize selection bias by ensuring that the study population was representative of the UK population in terms of geographic spread and sociodemographic characteristics. Advertisements were placed in both online and print media sources, including several different local and national newspapers to ensure data were captured from different sociodemographic populations.

\section{Statistical analysis}

All subjects who completed the questionnaire were included in the analysis. Subjects found not to have satisfied the protocol inclusion/exclusion criteria were excluded from the analysis. Analysis was carried out using SPSS version 20.0 (IBM Corporation, Armonk, NY, USA). Standard descriptive statistics such as mean and standard deviation were calculated; for non-normally distributed data, non-parametric statistics were used. Logistic regression models, adjusted for potential confounders (age, sex, socioeconomic status), were used to quantify the impact of treatment duration on adherence and persistence. Quality of life was a secondary endpoint. Key outcomes were compared between online and telephone-administered questionnaire responses to test for systematic differences (bias) resulting from the mode of administration. Missing data were not imputed.

\section{Results}

To recruit the 300 patients required, a total of 1,912 people responded to online or print advertisements designed to capture a representative sample of patients with different socioeconomic status. Of these, 1,059 were ineligible to participate and 545 did not manage to complete the survey. Reasons for noncompletion were variable with dropout occurring at different points in the questionnaire. The primary reasons for ineligibility were past treatment and timing of past treatment. A total of 305 eligible patients were recruited into the study and completed the questionnaire, $82 \%(n=250)$ of whom responded to local or national press advertisements.

Sociodemographic characteristics of the study population are reported in Table 1. AK lesions affected most body sites with the distribution of lesions as follows: $50 \%(n=152)$ of patients had lesions on the face or scalp only; $15 \%(n=46)$ had lesions on other body areas (eg, trunk, back, arms, legs, or feet) but not on the face or scalp; and $35 \%(n=107)$ had lesions on both the body and face or scalp. Table 2 shows the distribution of lesions by body site. More than half of patients had chronic AK lesions with 66\% $(n=202)$ diagnosed with their first lesion more than 1 year ago. At the time of recruitment, $43 \%(n=131)$ of patients were using topical therapy, and $40 \%(\mathrm{n}=120)$ had ceased therapy within the last 6 months. Sixty-eight percent of patients $(n=207)$ had visited a specialist in the previous 3 months. Around 50\% ( $\mathrm{n}=151)$ had experienced scarring resulting from their 
Table I Sociodemographic characteristics of the sample

\begin{tabular}{lll}
\hline Demographics & $\mathbf{n}=\mathbf{3 0 5}$ & $\%$ \\
\hline Sex & & \\
Male & 203 & 66.6 \\
Female & 102 & 33.4 \\
Age (years) & & \\
I9-25 & 12 & 3.9 \\
$26-30$ & 24 & 7.9 \\
$31-44$ & 14 & 4.6 \\
$45-55$ & 21 & 6.9 \\
$56-60$ & 26 & 8.5 \\
$61-65$ & 36 & 11.8 \\
$>65$ & 172 & 56.4 \\
Region & & \\
England & 243 & 79.7 \\
Wales & 12 & 3.9 \\
Scotland & 44 & 14.4 \\
Northern Ireland & 6 & 2.0 \\
Ethnicity & & \\
White & 297 & 97.4 \\
Black/Afro-Caribbean & 4 & 1.3 \\
Asian & 2 & 0.7 \\
Others (please specify) & 1 & 0.3 \\
Prefer not to answer & 1 & 0.3 \\
\hline
\end{tabular}

treatment. (This does not represent the true prevalence of scarring. Participants were purposefully recruited to ensure equal numbers of those who had and had not experienced scarring in order to examine the impact of previous scarring on adherence.)

\section{Patterns of use}

Non-adherence was derived from patient reports of patterns of use of topical therapies (when and how often they applied it). Non-adherence was defined as using more or less than the recommended dose, or more or less frequently than recommended according to the relevant summary of product characteristics. A total of $269(88 \%)$ patients were

Table 2 Distribution of AK lesions by body site: the number and percentage of patients with lesions at each body site and the median number of lesions at that site

\begin{tabular}{llllll}
\hline $\begin{array}{l}\text { Site of AK } \\
\text { lesions }\end{array}$ & $\mathbf{n}$ & $\%$ & Median & Min-Max & $\begin{array}{l}\text { Interquartile } \\
\text { range }\end{array}$ \\
\hline Scalp & 142 & 46.5 & 3.0 & $\mathrm{I}-50$ & $2-6$ \\
Face & 193 & 63.2 & 2.0 & $\mathrm{I}-5$ & $\mathrm{I}-5$ \\
Shoulders & 47 & $\mathrm{I} 5.4$ & 2.0 & $\mathrm{I}-\mathrm{I} 2$ & $\mathrm{I}-4$ \\
Back & 49 & $\mathrm{I} 6 . \mathrm{I}$ & 2.0 & $\mathrm{I}-\mathrm{I} 2$ & $\mathrm{I}-4$ \\
Trunk & 34 & $\mathrm{II} .2$ & 2.0 & $\mathrm{I}-30$ & $\mathrm{I}-4$ \\
Arms & 76 & 24.9 & 2.0 & $\mathrm{I}-90$ & $\mathrm{I} .25-9$ \\
Hands & 73 & 23.9 & 3.0 & $\mathrm{I}-30$ & $2-9$ \\
Legs & 54 & $\mathrm{I} 7.7$ & 3.0 & $\mathrm{I}-50$ & $\mathrm{I}-\mathrm{I} 3.5$ \\
Feet & $\mathrm{I} 5$ & 4.9 & 2.0 & $\mathrm{I}-30$ & $\mathrm{I}-\mathrm{I0}$ \\
\hline
\end{tabular}

Abbreviations: AK, actinic keratosis; Min, minimum; Max, maximum. either non-adherent, non-persistent, or both non-adherent and non-persistent. Sixty-three percent $(n=193)$ of subjects were non-adherent with their topical therapy for AK.

Thirty-one percent $(n=94)$ of patients had stopped using their treatment before the end of the recommended treatment period (non-persistent). Figure 1 shows the relationships between intentional (making a decision to use more or less than the recommended dose) and unintentional (forgetting doses) adherence and non-persistence (stopping treatment before the end of the course). Not all patients who were non-persistent were also non-adherent; some patients stopped taking their treatment before they had completed the recommended treatment period, but while they were using the medication, applied it with the recommended frequency in the recommended amounts.

The most recent treatment used by almost $50 \%(n=150)$ of patients had a recommended duration of 6-12 weeks, while $41 \%(n=126)$ used a treatment with the shortest recommended treatment duration of 3-4 weeks.

\section{Non-adherence, non-persistence and duration of treatment}

Rates of non-adherence increased with the duration of treatment with a significant increase when the treatment duration exceeded 5 weeks. Figure 2 shows rates of non-adherence by length of treatment period. Non-adherence (both intentional and unintentional) was twice as likely to occur with treatment durations greater than 4 weeks (adjusted OR: 2.2; CI 95\%: 1.3 to $3.6 ; P<0.01$, and OR: 2.6 ; CI 95\%: 1.5 to 4.3 ; $P<0.0001$ respectively). Rates of non-persistence showed a similar pattern with increasing treatment duration. Fourteen percent $(n=43)$ of patients were non-persistent with treatment with a 3-4 week treatment period, $21 \%(n=64)$ were

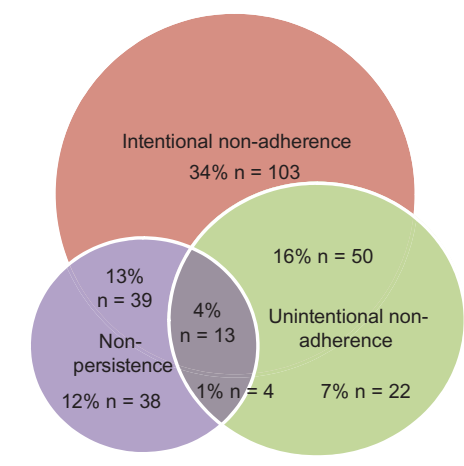

Figure I The relationships between intentional non-adherence (conscious choices to use medication in a way that differs from prescribed/recommended use), unintentional non-adherence (forgetting doses), and non-persistence (stopping treatment before completion of the recommended treatment period). 


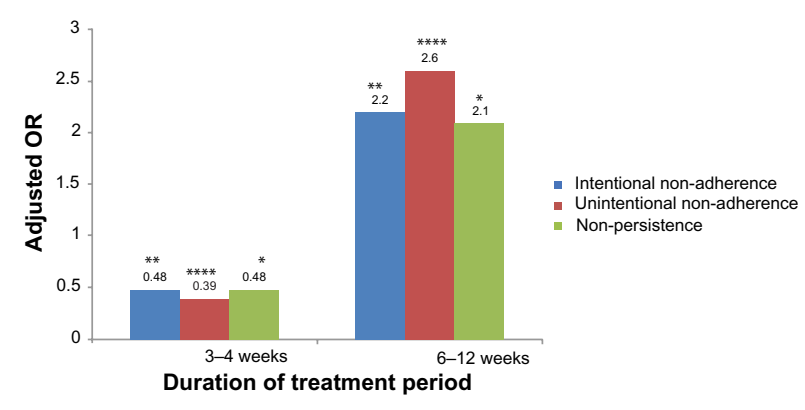

Figure 2 Adjusted odds ratios for intentional non-adherence, unintentional nonadherence, and non-persistence for treatment of different durations.

Notes: Statistical significance is represented by $* P<0.05 ; * * P<0.01$; $* * * P<0.00$ I; $* * * * * P<0.0001$.

Abbreviation: $O R$, odds ratio.

non-persistent with a 4-8 week treatment period, and $20 \%$ ( $n=61)$ were non-persistent with a 6-12 week treatment period (Figure 2).

Patient perceptions of the duration and intrusiveness of treatment also influenced adherence. Almost half of patients $(42 \%, \mathrm{n}=128)$ felt their treatment went on for too long and $74 \%(n=225)$ felt that treatment was too time consuming. Patients who felt that treatment went on for too long and that treatment was too time consuming to fit into their daily routine were more likely to be unintentionally non-adherent than adherent (adjusted OR: 1.2; CI 95\%: 1.1 to $1.3 ; P<0.0001$, and adjusted OR: 1.1 ; CI $95 \%$ : 1.0 to $1.8 ; P<0.05)$.

Duration of the treatment period was also associated with patients' perceptions of the efficacy of treatment; perceptions of efficacy decreased with increasing treatment duration $(P<0.0001)$ (Figure 3).

\section{Treatment outcomes and quality of life}

Less than half of patients $(45 \%, n=137)$ were concerned about the possibility of experiencing scarring from treatment or about the possibility of other side effects but those who were worried about scarring were more likely to be nonadherent than adherent (adjusted OR: 1.8; CI 95\%: 1.1 to 3.0; $P<0.05)$.

Patients who had experienced scarring as a result of treatment had poorer quality of life scores than those who had no scarring. The median Skindex 16 score for patients who had experienced scarring was 28.21, compared with 16.67 for patients who had no scarring, $P<0.001$ (higher scores indicate worse quality of life).

\section{Discussion}

This cross-sectional study examined adherence and persistence with topical treatments in patients with AK. Similar

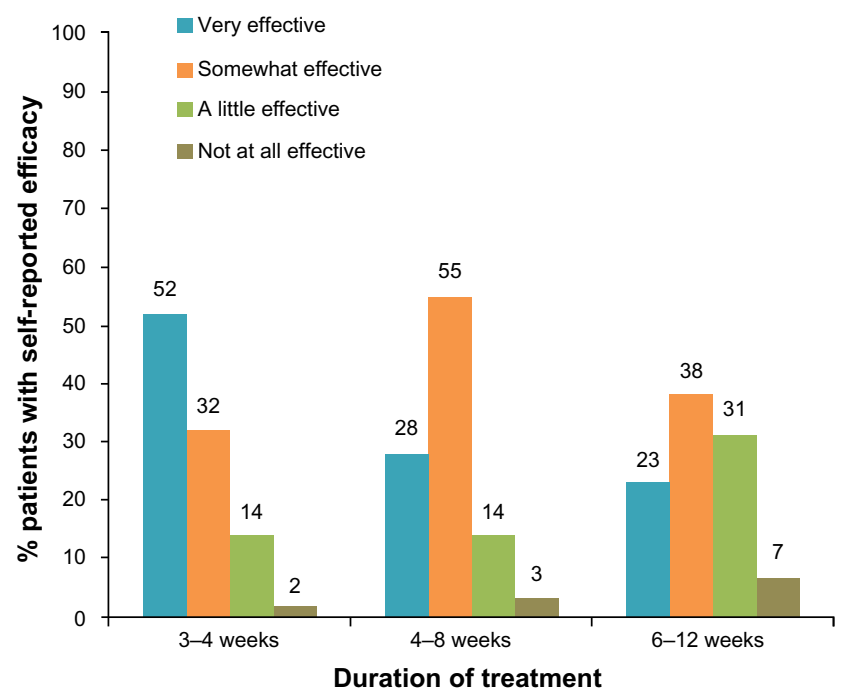

Figure 3 The impact of duration of treatment on patient perceptions of efficacy. Notes: Multiple regression analysis showed a significant decrease in perceived efficacy with increasing duration of treatment $(P<0.000 \mathrm{I})$

to published studies examining adherence with patientapplied topical therapy regimens in other skin disorders, ${ }^{5,10}$ rates of adherence and persistence in AK were found to be low and were much lower than those reported in a previous study in AK. ${ }^{8}$ Substantial proportions of patients with AK forgot to use their treatment or intentionally chose not to apply treatment according to product recommendations, and discontinued before the course was complete. Explanations for the lower levels of adherence in this study compared with the study reported by Yentzer et al, ${ }^{8}$ may include the larger sample size in this study with broader inclusion criteria; patients had lesions on their trunk and/ or extremities as well as their face and scalp and different topical therapies. In addition, patients in the study by Yentzer et al were assessed prospectively at regular intervals during the treatment period and, although adherence was not specifically addressed during those assessments, the fact that they were being actively followed-up may have prompted greater adherence.

In line with the literature, duration of treatment and perceived ease of use of treatment affected adherence and persistence in patients with AK. Duration of treatment was shown to be a strong predictor of non-adherence and non-persistence; both non-adherence and non-persistence increased with increasing duration of treatment.

Quality of life in patients with AK was higher posttreatment for all patients irrespective of whether or not they had experienced LSR or scarring and patients without scarring or LSR showed a consistently higher quality of life in all dimensions (emotional, physical, and functioning) than 
those who had experienced scarring or LSR. These findings demonstrate that there is a clear "global" benefit in terms of quality of life associated with treating AK, even in those patients who have experienced LSR or scarring.

A limitation of this study was the use of patient selfreport to capture information on adherence. Studies have consistently shown that patient-reported adherence is higher than objective measures of adherence. ${ }^{15-22}$ The difficulty is in finding appropriate and acceptable objective measures of adherence for patient-applied topical treatments. Measuring the used weight (of tubes or other containers) is often used as a more objective measure of adherence, although it tells us nothing about how the medication was used, when it was applied, to what it was applied, and how much was applied to each area. In this study we limited the bias inherent in patient self-report by asking patients to describe how and when they used their medication without any reference to whether that was what the doctor had recommended. This approach has been used to capture information on patterns of use of medication from which rates of adherence have been calculated across a number of therapy areas.

A further limitation of the study was the lack of confirmed diagnosis of AK or treatment. Some patients may have participated in the study who had other conditions. However, the screening interviews to assess eligibility were detailed and rigorous, to minimize the potential for bias.

Using different methods of data collection could also potentially bias the results, with patients who complete a telephone administered questionnaire giving different responses to key questions about adherence from those completing the questionnaire online. Our researchers were specifically trained to try to minimize this bias. Moreover, the way that the questions were framed (around how patients used their topical therapies) was designed to remove any impression of a right or wrong way of using treatment, to reduce the over-reporting of adherence that so frequently happens in self-report. Key outcomes were compared between online and telephone administered questionnaires and no significant differences were found, suggesting that the differences in methods of data collection did not bias the results.

\section{Conclusion}

This study found high rates of non-adherence and nonpersistence to patient-applied topical therapy in the UK. These findings are consistent with published studies which report high rates of non-adherence with patient-applied topical therapy regimens for other skin conditions. The duration of treatment is a significant factor influencing non-adherence and non-persistence, with significantly higher rates of both when the treatment duration exceeds 4 weeks. The findings of this study provide some insight into potential strategies for improving adherence to patient-applied topical treatment regimens. Treatment-related factors such as ease of use and duration of treatment may be amenable to change through interventions that manage patient expectations and provide support during the treatment period. Adherence may be improved with simplification of the treatment regimen, less frequent use, and shorter treatment duration.

\section{Disclosure}

This research was funded by Leo Pharma. The funder was involved in the study design and manuscript preparation but all data collection and data analysis were performed independently. Dr Simon Zokaie is an employee of Leo Pharma. Dr Alison Carr is a Director of Hamell, who were contracted and paid by Leo Pharma to conduct the research. The authors report no other conflicts of interest in this work.

\section{References}

1. de Berker D, McGregor JM, Hughes, et al; British Association of Dermatologists Therapy Guidelines and Audit Subcommittee. Guidelines for the management of actinic keratoses. Br J Dermatol. 2007;156(2): 222-230.

2. Berman B, Bienstock L, Kuritzky L, Mayeaux EJ Jr, Tyring SK; Primary Care Education Consortium; Texas Academy of Family Physicians. Actinic keratoses: sequelae and treatments. Recommendations from a consensus panel. J Fam Prac. 2006;55(Suppl):1-8.

3. Moy RL. Clinical presentation of actinic keratoses and squamous cell carcinoma. J Am Acad Dermatol. 2000;42(1 Pt 2):8-10.

4. Glogau R. The risk of progression to invasive disease. $J$ Am Acad Dermatol. 2000;42(1 Pt 2):23-24.

5. Devaux S, Castela A, Archier E, et al. Adherence to topical treatment in psoriasis: a systematic literature review. J Eur Acad Dermatology and Venereol. 2012(Suppl 3):61-67.

6. Lee IA, Maibach HI. Pharmionics in dermatology: a review of topical medication adherence. Am J Clin Dermatol. 2006;7(4):231-236.

7. Feldman SR, Horn EJ, Balkrishnan R, et al; International Psoriasis Council. Psoriasis: improving adherence to topical therapy. J Am Acad Dermatol. 2008;59(5):1009-1016.

8. Yentzer B, Hick J, Williams L, et al. Adherence to a topical regimen of 5 -fluorouracil, $0.5 \%$ cream for the treatment of actinic keratosis. Arch Dermatol. 2009;145(2):203-205.

9. Dreno B, Thiboutot D, Gollnick H, et al; Global Alliance to Improve Outcomes in Acne. Large-scale worldwide observational study of adherence with acne therapy. Int J Dermatol. 2010;49(4):448-456.

10. Zaghloul SS, Goodfield MJD. Objective assessment of compliance with psoriasis treatment. Arch Dermatol. 2004;140(4):408-414.

11. Brown KK, Rehmus WE, Kilmball AB. Determining the relative importance of patient motivations for nonadherence to topical corticosteroid therapy in psoriasis. J Am Acad Dermatol. 2006;55(4):607-613.

12. Lott R, Taylor SL, O’Neill JL, Krowchuk DP, Feldman SR. Medication adherence among acne patients: a review. J Cosmet Dermatol. 2010;9(2):160-166.

13. Carr AJ, Hughes RA, Vincent K, Carr M, Thwaites C. The impact and implications of new treatments in arthritis: validation of a questionnaire to measure the real life effectiveness of medication. Rheumatology 2004;43(suppl):ii83 
14. Carr AJ, Thompson PW, Cooper C. Factors affecting adherence and persistence to bisphosphonate therapy in osteoporosis: a cross-sectional study. Osteoporosis International 2006;17(11):1638-1644

15. Carroll CL, Feldman SR, Camacho FT, et al. Adherence to topical therapy decreases during the course of an 8-week psoriasis clinical trial: commonly used methods of measuring adherence to topical therapy overestimate actual use. J Am Acad Dermat. 2004;51(2):212-216.

16. Straka RJ, Fish JT, Benson SR, Suh JT. Patient self-reporting of compliance does not correspond with electronic monitoring: an evaluation using isosorbide dinitrate as a model drug. Pharmacotherapy. 1997; 17(1):126-132.

17. Llabre MM, Weaver KE, Duran RE, Antoni MH, McPherson-Baker S, Schneiderman N. A measurement model of medication adherence to highly active antiretroviral therapy and its relation to viral load in HIV positive patients. AIDS Patient Care STDS. 2006;20(10):701-711.

18. Lee MS, Lee HY, Kang SG, et al. Variables influencing antidepressant medication adherence for treating outpatients with depressive disorders. J Affective Disorders. 2010;123(1-3):216-221.
19. Bell DJ, Kapitao Y, Sikwese R, van Oosterhout JL, Lalloo DG Adherence to antiretroviral therapy in patients receiving free treatment from a government hospital in Blantyre, Malawi. J Acquir Defic Syndr. 2007;45(5):560-563.

20. Shi L, Liu J, Walker P, Kalsekar A, Pawaskar M. Correlation beween adherence rates measured by MEMS and self-reported questionnaires: a meta-analysis. Health Qual Life Outcomes. 2010;8:99.

21. Fletcher CV, Testa MA, Brundage RC, et al. Four measures of antiretroviral medication adherence and virologic response in AIDS clinical trials group study 359. J Acquir Immune Defic Syndr. 2005; 40(3):301-306.

22. Hamilton GA. Measuring adherence in a hypertension clinical trial. Eur J Cardiovasc Nurs. 2003;2(3):219-228.

23. Chren MM, Lasek RJ, Sahay AP, Sands LP. Measurement of properties of Skindex-16, a brief quality-of-life measure for patients with skin diseases. J Cutan Med Surg. 2001;5(2):105-110.
Patient Preference and Adherence

\section{Publish your work in this journal}

Patient Preference and Adherence is an international, peer-reviewed, open access journal focusing on the growing importance of patient preference and adherence throughout the therapeutic continuum. Patient satisfaction, acceptability, quality of life, compliance, persistence and their role in developing new therapeutic modalities and compounds to

\section{Dovepress}

optimize clinical outcomes for existing disease states are major areas of interest. This journal has been accepted for indexing on PubMed Central. The manuscript management system is completely online and includes a very quick and fair peer-review system. Visit http://www.dovepress.com/ testimonials.php to read real quotes from published authors.

Submit your manuscript here: http://www.dovepress.com/patient-preference-and-adherence-journal 